\title{
Neutron star equation of state and uncertainty on the radius determination
}

\author{
Morgane Fortin \\ Nicolaus Copernicus Astronomical Center of the Polish Academy of Sciences, Bartycka 18, \\ Warsaw 00-716, Poland \\ email: fortin@camk.edu.pl
}

\begin{abstract}
Simultaneous measurements of the radius and mass of neutron stars (NSs) are expected from the new generation of X-ray telescopes, potentially constraining the NS equation of state (EoS). However using 'non-unified' EoSs with the ones for the core and the crust not based on the same nuclear model can introduce an uncertainty on the radius as large as the precision expected from these instruments. I present two solutions to this problem: a large collection of unified EoSs and an approximate and yet precise approach that, with no need of a crust EoS, provides the relation between the NS mass and radius. I discuss correlations between the NS radius and nuclear parameters, possibly allowing to constrain the NS radius with experiments on Earth. Finally, I show that in spite of the observation of massive NSs, one can not exclude that hyperons appear at high densities in NSs due to the scarcity of the available experimental data.
\end{abstract}

Keywords. stars: neutron, pulsars: general, equation of state

\section{Introduction}

NSs have been observed for the first time 50 years ago and yet relatively little is known about the properties of matter inside them. The EoS which describes the properties and composition of matter in their interior is one of their many mysteries for two reasons: the modeling of a many-body system of strongly interacting particles as found inside NSs is a complex and unsolved problem (see e.g., Haensel et al. 2007; Oertel et al. 2017) and neutron-rich cold dense matter is not accessible in terrestrial laboratories. However thanks to the determination of some astrophysical NS properties such as their mass $M$ and radius $R$ one may constrain the EoS and thus potentially unveil the properties of NS matter. For the time being, the only constraint comes from the observation of the two pulsars with masses close to $2 M_{\odot}$ (Antoniadis et al. 2013; Fonseca et al. 2016). Simultaneous determinations of the mass and radius of a number of NSs thus providing further and more stringent constraints are expected in the near future thanks to the newly-operating NICER (Arzoumanian et al. 2014) and Athena (Motch et al. 2013) missions.

\section{Non-unified equations of state and radius uncertainty}

The NS interior consists of two main parts. The upper one, the crust, is composed of a lattice of nuclei, permeated by a gas of electrons and of free neutrons at high density. At the transition between the crust and the central part of the NS, the core, at a density $n_{\text {cc }} \simeq 0.5 n_{0}$, where $n_{0}=0.16 \mathrm{fm}^{-3}$ is the nuclear saturation density, the nuclei disappear. The core is then formed of free nucleons, in addition to electrons and muons, and possibly more exotic forms of matter like hyperons (baryons like the neutron and the proton, but with a least one strange quark) at high densities. 

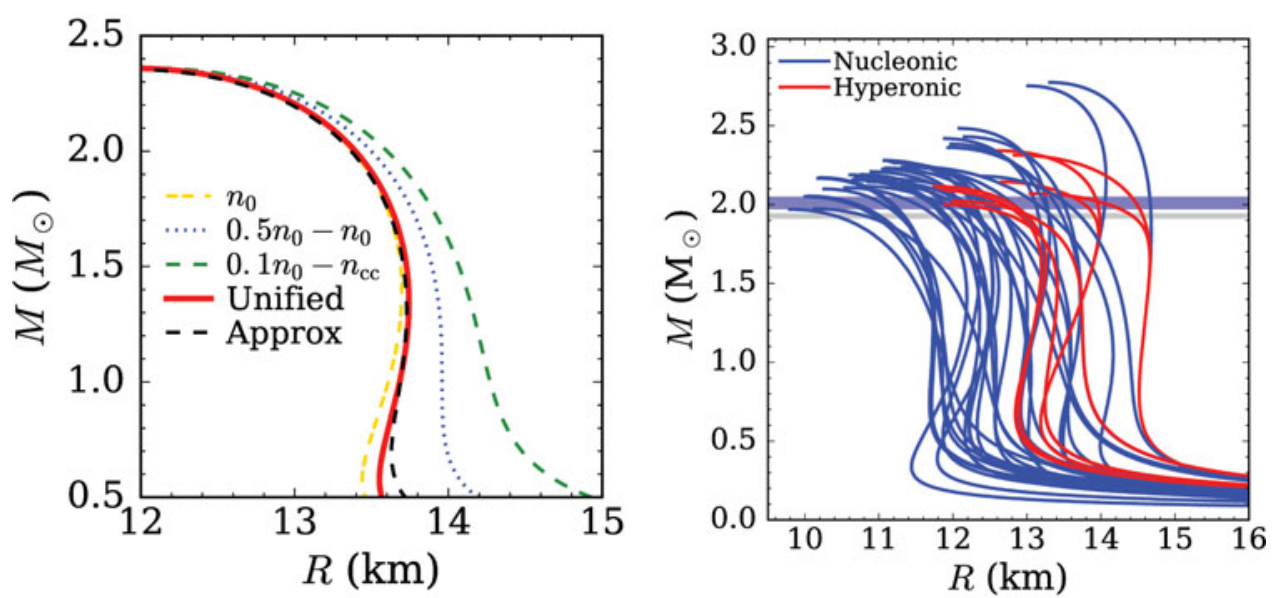

Figure 1. Left: relations between the NS mass and radius obtained with different gluing procedures employed in the literature, using the GM1 EoS for the core and the non-consistent EoS from Douchin \& Haensel (2001) for the crust. Crust glued to the core at the density $n_{0}$ (labeled $n_{0}$ ), crust EoS up to $0.5 n_{0}$ and core EoS from $n_{0}$ (label $0.5 n_{0}-n_{0}$ ), and crust up to $0.1 n_{0}$ and core from the density $n_{\mathrm{cc}}$ : the theoretically calculated core-crust transition for the GM1 $\operatorname{EoS}\left(0.1 n_{0}-n_{\mathrm{cc}}\right)$. The 'unified' curve is obtained using unified core and crust EoSs from Fortin et al. (2016) and the 'approx' relation using the approximate approach from Zdunik et al. (2017) presented in Sect. 3. Right: $M-R$ relations obtained with the unified EoSs calculated in Fortin et al. (2016) for a purely nucleonic core (in blue) and for a core with a transition to hyperonic matter (in red). All EoSs are consistent with $2 M_{\odot}$ NSs.

Since the crust is non-uniform as it is composed of atomic nuclei, calculating its EoS is much less straightforward than for the homogeneous core. This is why there are much less crust EoSs available compared to core ones. In particular very few unified EoSs, for which the properties of both the core and the crust have been calculated based on the same nuclear model, have been developed. However an EoS describing the entire NS interior, core and crust, is necessary to calculate for example the relation between the NS mass and radius. Consequently a widely-used approach consists in using two EoSs, one for the crust and another one for the core based on different nuclear models, and connecting (or 'gluing') them together. However, as shown in Fortin et al. (2016) and in the left panel of Fig. 1, the various gluing procedures reported in the literature result in differences in the radius for a $1.0 M_{\odot} \mathrm{NS}$ which can be as large as $\sim 1 \mathrm{~km}$ ! This is problematic as it is of the same order as the expected precision on the radius measurement from the new generation of X-ray telescopes (NICER, Athena, ... ). Thus this problem of the core-crust gluing has to be solved or at least handle, so that constraints on the EoS can be correctly obtained from future combined NS mass-radius determinations.

\section{Unified equations of state and approximate approach to the crust}

I present here two strategies to tackle the problem posed by the gluing of a crust EoS to one for the core.

The first one consists in using unified EoSs for which the core and crust EoSs are obtained using the same nuclear model. In Fortin et al. (2016) a set of 33 EoSs with a purely nucleonic core (ie. with only neutrons, protons, electrons, and muons), and 15 hyperonic ones (with hyperons appearing at high density) is presented. They are all consistent with the existence of $2 M_{\odot}$ NSs as shown in the right panel of Fig. 1 and cover a large part of the $M-R$ plane. All these EoSs are available in the form 
of tables as a supplemental material to Ref. Fortin et al. (2016) . In addition the 33 nuclear models are confronted to a collection of modern nuclear constraints both from experiments (constraints on the nuclear parameters) and theoretical calculations (for pure neutron matter for densities below $n_{0}$ ). A set of 4 nuclear models, 4 nucleonic and 4 hyperonic EoSs are then selected. For reference, they give $R_{1.4}=13.10 \pm 0.65 \mathrm{~km}$ for the radius of a $1.4 M_{\odot} \mathrm{NS}$.

The second strategy presented in Zdunik et al. (2017) can be used if the crust EoS is not available for the nuclear model used for the core EoS. It relies on an approximate approach to the NS crust and consists in rewriting the TOV equations for hydrostatic equilibrium in General Relativity assuming that the mass of the crust is small compared to the total mass of the star. The assumption is correct for masses typically larger than $1 M_{\odot}$. One then obtain the following relation between the radius of the core $R_{\text {core }}$ and the total radius of the NS:

$$
\frac{\sqrt{1-2 G M / R c^{2}}}{\sqrt{1-2 G M / R_{\mathrm{core}} c^{2}}}=\frac{\mu_{\mathrm{cc}}}{\mu_{0}}
$$

With this equation, one can then obtain the relation between the mass and radius of a NS using only an EoS for the core. In other words, with this approach, no crust EoS is required. First one solves the TOV equations using only the core EoS, for densities larger than $n_{\text {cc }}$ (see below) and thus obtains the relation between $M$ and $R_{\text {core }}$. Then the relation between the total radius of the star $R$ and $M$ can be simply obtained using Eq. (3.1). Two quantities enter the latter: 1) the baryon chemical potential at zero pressure $\mu_{0}$. For a non-accreted crust it corresponds to the one of ${ }^{56} \mathrm{Fe}$ as the minimum of energy is obtained for this species: $\mu_{0}=930.4 \mathrm{MeV}$ (Haensel et al. 2007); 2) the baryon chemical potential at the core-crust transition $\mu_{\mathrm{cc}}$. The location of the transition is usually not known for a given core EoS. However for a large set of EoSs Ducoin et al. (2011) show that $0.38 \leqslant n_{\mathrm{cc}} / n_{0} \leqslant 0.63$. Consequently, taking $n_{\mathrm{cc}}=0.5 n_{0}$ appears reasonable. Then the chemical potential at the transition is given by $\mu_{\mathrm{cc}}=\left(P\left(n_{\mathrm{cc}}\right)+\rho\left(n_{\mathrm{cc}}\right)\right) / n_{\mathrm{cc}}$, where $P\left(n_{\mathrm{cc}}\right)$ and $\rho\left(n_{\mathrm{cc}}\right)$ are the pressure and mass-energy density obtained using the core EoS. For $M>1 M_{\odot}$ the approximate approach gives values of $R$ with a very good precision: $<0.2 \%$. An example is plotted in Fig. 1 (label 'approx') for the GM1 EoS and the theoretically calculated core-crust transition density $n_{\mathrm{cc}}=0.41 n_{0}$. Similar simple and yet very precise formulas applicable for a NS with an accreted crust and for the crust thickness are also obtained in Zdunik et al. (2017).

\section{Nuclear parameters and radii}

Nuclear parameters that are properties describing the NS EoS can be measured in laboratory and potentially constrain the NS radius, complementing astrophysical observations. Indeed correlations between various nuclear parameters and $R$ have been investigated extensively. For example, in line with numerous previous works, Fortin et al. (2016) confirm the existence of a linear correlation between $L$, the so-called slope of the symmetry energy at saturation, and the NS radius using 33 unified EoSs. The existence of a strong correlation between $R$ and a linear combination of $L$ and the slope $M$ of the incompressibility $K$ evaluated at saturation is presented in Alam et al. (2016) employing 44 EoSs all consistent with $2 M_{\odot}$ NSs. This correlation is found to be almost independent of the NS mass. Since $L$ and $M$ are experimentally constrained from measurements of various nuclear properties, one then obtain $R_{1.4}=11.09-12.86 \mathrm{~km}$. 


\section{Experimentally calibrated hyperonic EoSs}

Relatively little is known about hyperons (Gal et al. 2016) although they were discovered in laboratory in the early 1950s and are studied experimentally since then. The binding energy of $\sim 50$ hypernuclei (i. e. nuclei with at least an hyperon) has been measured. In the late 1950s it was suggested that hyperons could be present in NS cores. However the existence of massive NSs can appear difficult to reconcile with the presence of hyperons in the NS core. Indeed hyperons usually lead to the softening of the EoS (smaller pressure at high density than for a nucleonic EoS) and thus to a lower value of the NS maximum mass. This is the so-called hyperon puzzle.

In Fortin et al. (2017), hyperonic EoSs are obtained for 6 nuclear models. The couplings for hyperons that enter the EoS model are calibrated to reproduce all the available but scarce experimental data on hypernuclei properties. The remaining uncertainties on the hyperon properties are also taken into account and shown to lead to a large range of possible maximum masses for hyperon NSs, within $0.3 M_{\odot}$. In conclusion the existence of $2 M_{\odot}$ NSs does not rule out the presence of hyperons in their core. More measurements of the hypernuclei properties are need to solve the hyperon puzzle.

\section{Conclusion}

Accurate constraints on the properties of NS matter thanks to incoming joint measurements of the mass and radius of a number of NSs from the new generation of X-ray satellites can only be obtained if a careful treatment of the NS EoS at the core-crust transition is used. This can be achieved by employing unified EoSs, based on the same nuclear model for the core and the crust, such as the ones presented in Fortin et al. (2016), or using the approximate and yet very accurate approach to the NS crust developed in Zdunik et al. (2017). Additional constraints on the NS radius can also be obtained using measurements of nuclear parameters from laboratory experiments (Fortin et al. 2016; Alam et al. 2016). Finally, as shown in Fortin et al. (2017), more measurements of the hyperon properties are necessary in order to confirm or exclude the presence of hyperons inside the core of massive NSs.

This work was supported by the Polish National Science Centre (NCN) grant UMO2014/13/B/ST9/02621 and the New-Compstar COST Action MP1304.

\section{References}

Alam, N., Agrawal, B. K., Fortin, M., et al. 2016, Phys. Rev. C, 94, 052801

Antoniadis, J., Freire, P. C. C., Wex, N., et al. 2013, Science, 340, 448

Arzoumanian, Z., Gendreau, K. C., Baker, C. L., et al. 2014, Proc. SPIE, 9144, 914420

Douchin, F. \& Haensel, P. 2001, A\& A, 380, 151

Ducoin, C., Margueron, J., Providência, C., \& Vidaña, I. 2011, Phys. Rev. C, 83, 045810

Fonseca, E., Pennucci, T. T., Ellis, J. A., et al. 2016, ApJ, 832, 167

Fortin, M., Providência, C., Raduta, A. R., et al. 2016, Phys. Rev. C, 94, 035804

Fortin, M., Avancini, S. S., Providência, C., \& Vidaña, I. 2017, Phys. Rev. C, 95, 065803

Gal, A., Hungerford, E. V., \& Millener, D. J. 2016, Rev. of Modern Phys., 88, 035004

Haensel, P., Potekhin, A. Y., \& Yakovlev, D. G. 2007, Astrophysics and Space Science Library, 326

Motch, C., Wilms, J., Barret, D., et al. 2013, arXiv:1306.2334

Oertel, M., Hempel, M., Klähn, T., \& Typel, S. 2017, Rev. of Modern Phys., 89, 015007

Zdunik, J. L., Fortin, M., \& Haensel, P. 2017, A\& A, 599, A119 